\title{
A Comparative Clinical Demonstration of the Spreadability of Tazarotene Lotion $0.045 \%$ versus Trifarotene Cream 0.005\%
}

Zoe D Draelos, MD'; Emil A Tanghetti, MD²

Dermatology Consulting Services, PLLC, High Point, North Carolina; ${ }^{2}$ Center for Dermatology and Laser Surgery, Sacramento, CA

\section{SYNOPSIS}

- The ability of a topical medication to spread is an important

parameter, since only the thinnest layer of medication contacting the skin is physiologically active

A thinner film is just as effective as a thicker film from an efficacy standpoint, but a thinner film will spread farther-exhibiting superior spreadability and increasing the number of applications while decreasing the cost per application

- From a rheological perspective, products exhibiting low yield stress and lower intrinsic viscosity will have better spreadability and require less effort to spread at the surface of the skin ${ }^{1,2}$ - Yield stress is the minimum force required to make a structured fluid flow

Viscosity describes a fluid's resistance to flow (eg, the "thickness" of a fluid)

OBJECTIVE

- To compare the spreadability of two topical formulations: tazarotene $0.045 \%$ polymeric emulsion lotion versus trifarotene $0.005 \%$ cream

To relate the rheological profile of topical products to their spreadability

METHODS

- This double-blind, split-body study enrolled male or female adults $\geq 18$ years of age with normal back ski

- Participants, who provided written informed consent, were assessed for limited back hair which would prevent application of the study products

Tazarotene $0.045 \%$ lotion was applied to one randomized half of the back and trifarotene $0.005 \%$ cream was applied to the opposite randomized half of the back (Figure 1)

- The back was divided at the vertebral column into right and left - Drugs were randomized for right or left application; however, the left back product was always pigmented blue and the right back product was always pigmented green. One toothpick tip of blue or green food-coloring gel was used to pigment the drugs
- The blinded dermatologist investigator was presented with $0.1 \mathrm{cc}(0.1 \mathrm{~mL})$ of each of the drugs for application by the unblinded coordinator

Two $10 \mathrm{~cm}$ wide application areas were marked with a gentian violet marker, one on each side of the back; this mark defined the lateral bounds over which the lotion or cream

The investigator applied the products with a gloved hand to obtain an even film moving study product down the back until it would no longer spread

The lower extent of the study product application was marked with a gentian violet marker and measured in centimeters

A two-tailed Student's t-test was used to assess the spreadability data

FIGURE 1. Study Schematic

tazarotene $0.045 \%$ lotion and randomized 1:1
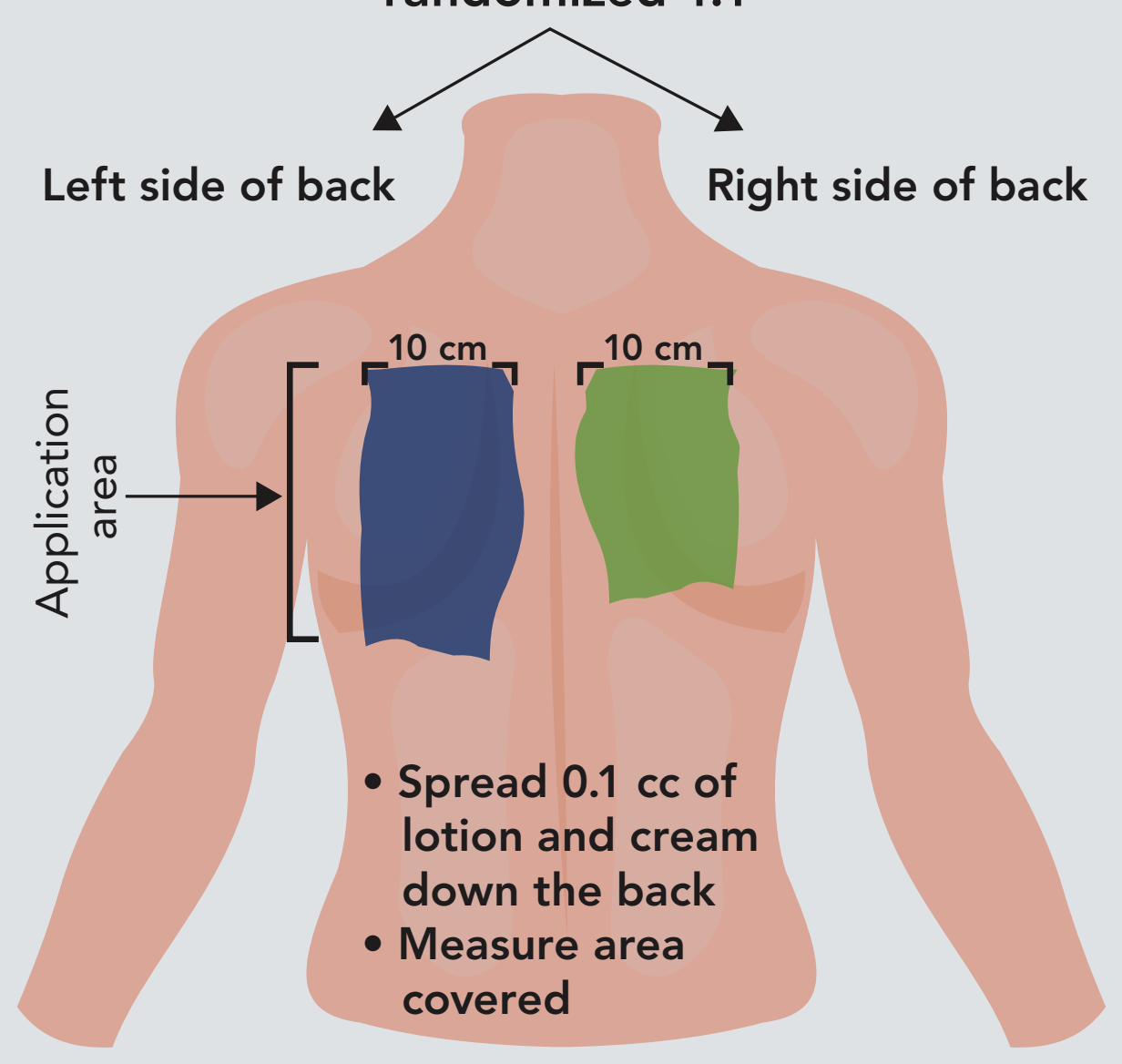

RESULTS

- A total of 30 participants were included in the study

- Participants ranged from 18 to 59 years of age: 26 (87\%)

were female

Tazarotene $0.045 \%$ lotion spread over an average area measuring $10 \mathrm{~cm} \times 16.70 \mathrm{~cm}\left(167.0 \mathrm{~cm}^{2}\right)$ while the trifarotene $0.005 \%$ cream spread over an average area measuring $10 \mathrm{~cm} \times 13.03 \mathrm{~cm}\left(130.3 \mathrm{~cm}^{2} ; P<0.001\right.$; Figure 2) - No adverse reactions or adverse events occurred during the conduct of the study

FIGURE 2. Mean Spreadability of Tazarotene $0.045 \%$ Lotion and Trifarotene $0.005 \%$ Cream $(\mathrm{N}=30)$

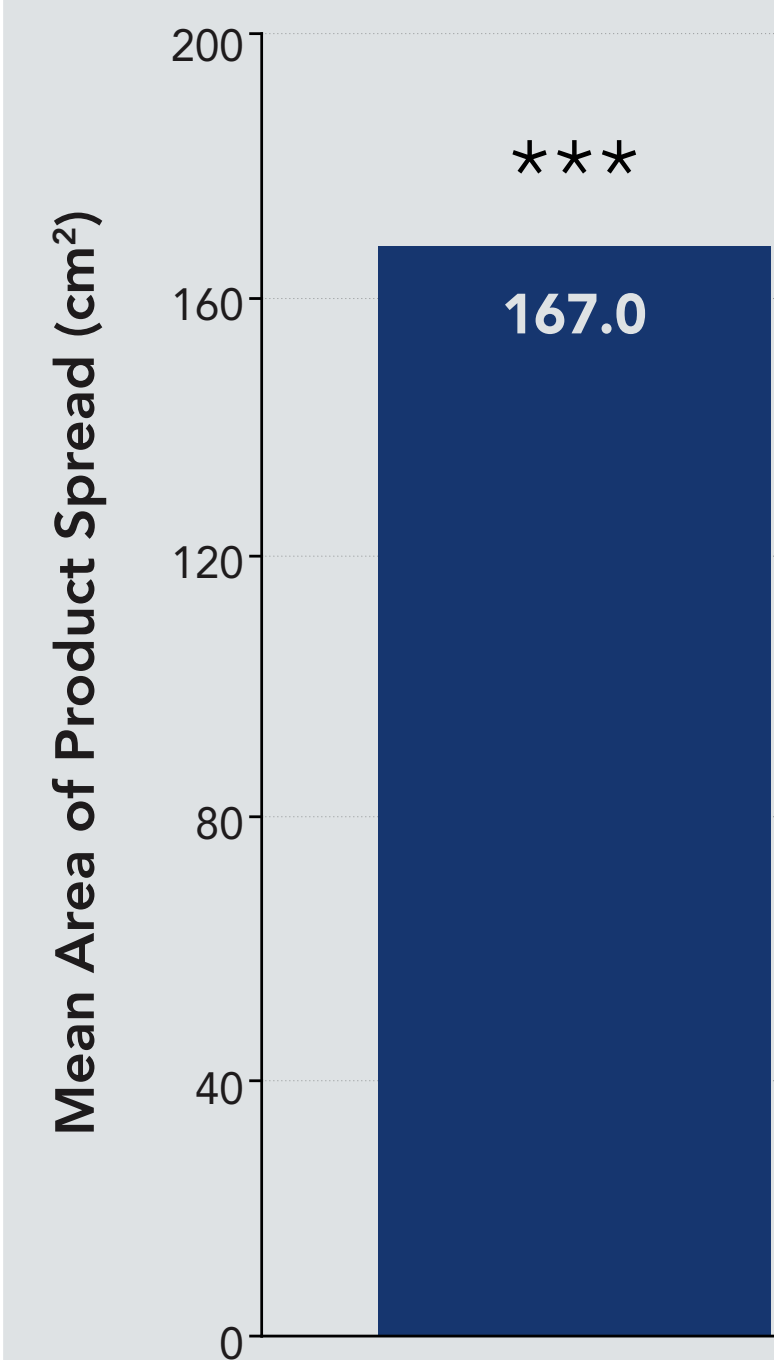

Tazarotene $0.045 \%$ Lotion

\section{3}

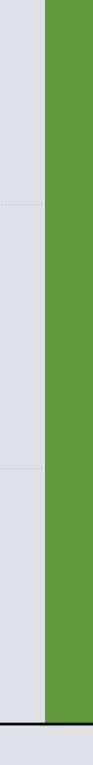

Trifarotene
FIGURE 3 Spreadability of Tazarotene $0.045 \%$ Lotion and Trifarotene $0.005 \%$ Cream on a Participant

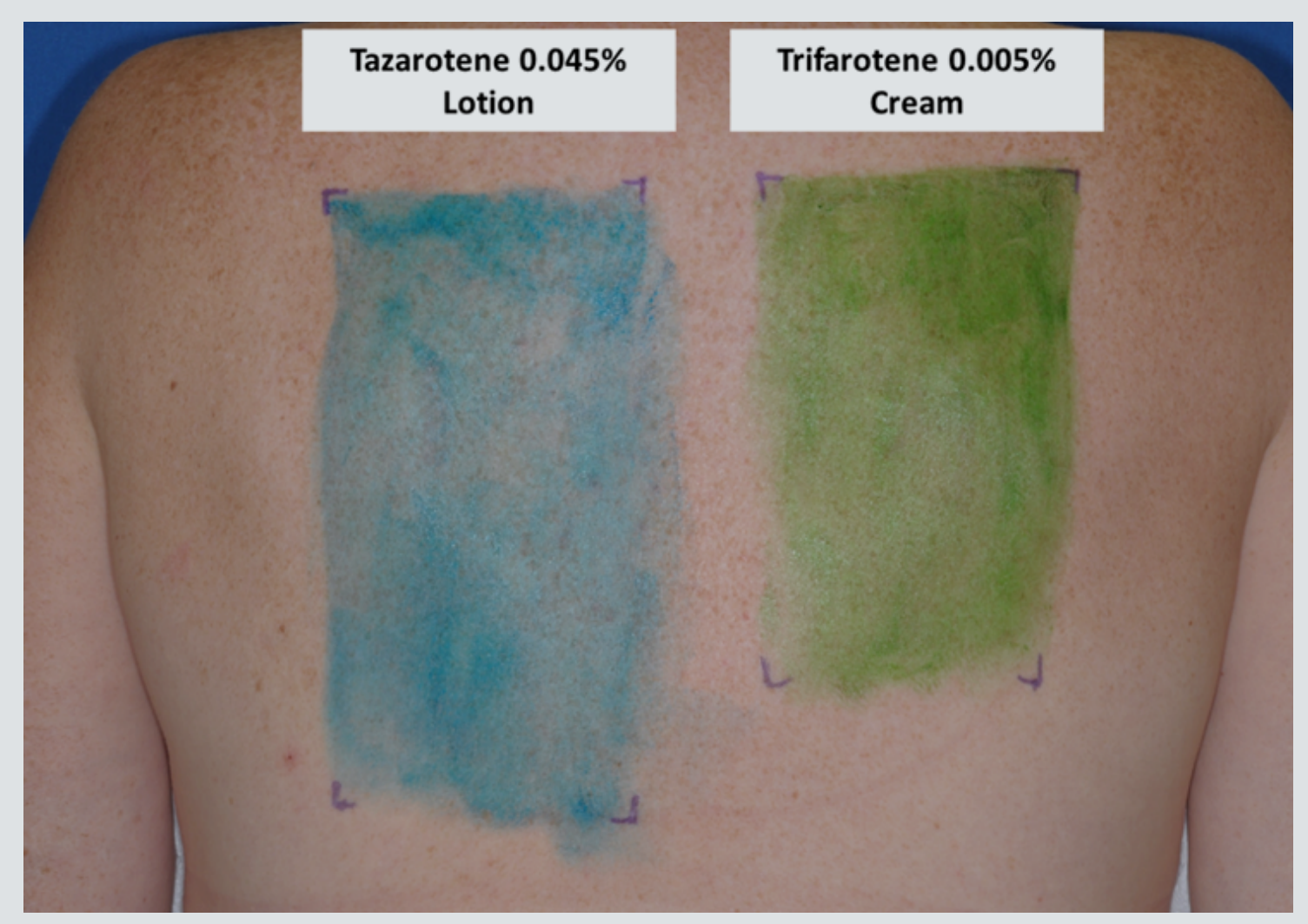

\section{CONCLUSIONS}

The tazarotene $0.045 \%$ lotion spread on average 36.7 square centimeters farther than the trifarotene $0.005 \%$ cream

- These results are supported by the differences in the rheological profiles of the two products, in which

tazarotene lotion exhibits lower yield stress and lower intrinsic viscosity versus trifarotene $\mathrm{cream}^{3}$

REFERENCES

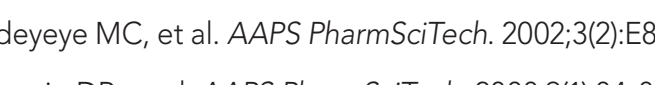

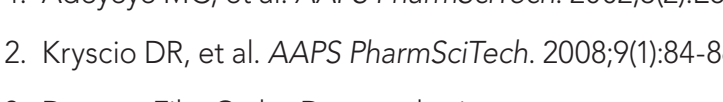

AUTHOR DISCLOSURES

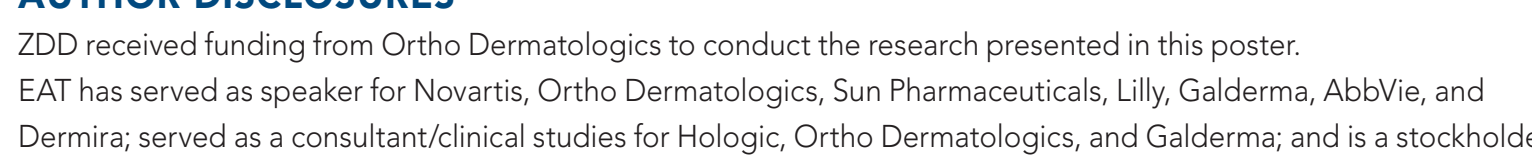

